\title{
Patient-Centered Surgical Care Meets the Social Determinants of Health
}

\author{
Michael Williams ${ }^{1}$
}

Accepted: 5 September 2020/Published online: 25 September 2020

(C) Société Internationale de Chirurgie 2020

In August 2020, the Centers for Medicare and Medicaid services (CMS), a division of the Department for Health and Human Services in the United States government, proposed reimbursement cuts of between 7 and 9\% for outpatient surgical care, including general surgery-specific procedures such as cholecystectomy [1]. If enacted, this new Fee Schedule may well have a chilling effect on the utilization of elective surgical procedures in the Medicare population due to cost shifts that are unaffordable for patients. It can easily be imagined that patients will therefore choose to delay care until symptoms are so severe that urgent and emergent procedures are required rather than on an elective basis. Secondary victims of this potential shift in utilization of surgical care will be those patients who are either uninsured or currently qualify for their state's Medicaid program, which provides health insurance for low-income adults and children. Combined with the ongoing COVID-19 pandemic, increasing pressure will be placed on healthcare systems to provide care for the uninsured in America. Both of these facts amplify the importance of the critical work by Hatton et al., "Timely Care is Patient-Centered Care for Patients with Acute Cholecystitis at a Safety-Net Hospital" [2].

The authors have taken an important step in reframing the discussion of surgical care and its value, from the $p a$ tient's perspective, differing from the preponderance of the literature which often focuses on either physician or health system-centric perspectives, goals and outcomes. By using qualitative methods including structured and semi-structured interviews, the authors have added to our knowledge

Michael Williams

MDW9G@hscmail.mcc.virginia.edu

University of Virginia Health System, Charlottesville, USA base considerably. By identifying, in rank order from the study participants' perspective, patient-specific and $p a$ tient-centric goals of care including focusing on such determinants of health as Time-to-Return-to-Work, among a population for whom loss of work productivity is often associated with termination of employment, the authors have elegantly re-examined the question of value-based care, through an equity lens.

When analyzed in the context of a US healthcare system that operates as "Big Business" while simultaneously being a fundamental necessity for the basic functioning of our society, the work by Hatton et al. becomes even more impactful. With health systems and other systems of care under increasing pressure to both contain costs and continually improve quality, papers such as this that focus on Safety-Net Hospitals and health systems are crucial in our shared efforts to realize an equitable healthcare system in this country. By "flipping the script" and examining length of stay (LOS) from the patient's perspective, not in days but hours, while redefining "Timeliness" from the same perspective, the authors give us insight into what patients value as compared to CMS" "Two Midnight Rule" per se. Hatton et al. have further validated the value proposition that a fully staffed Emergency General Surgery service, providing 24-h/day surgical care, regardless of payer status, adds value to both health systems and (most importantly) patients, particularly those who face economic or other barriers to healthcare access. It is troubling that several study participants reported that they had had symptoms for "multiple years." This, in spite of the fact that a wealth of the literature, cited by the authors, clearly supports firstpresentation cholecystectomy for patients with acute cholecystitis. It is my hope that surgeons, healthcare administrators and health systems as a whole will 
incorporate patient-centered, social determinant-guided care into their business operations.

Hatton et al. have clearly demonstrated the value of such an approach not only from a business perspective, (firstpresentation cholecystectomy with $<24 \mathrm{~h}$ LOS and no repeated visits to the ED for symptomatic patients who lack insurance being the most cost-effective approach for both patients and healthcare systems) but crucially, from that of the population of patients who will continue to, and perhaps increasingly, only have their care needs met through an increasingly fragile Healthcare Safety Net. A US healthcare system that is not just profitable but also Just should be the goal of all surgeons and all physicians, indeed all of us who serve in the healthcare arena. Hatton and co-authors have shown us that by thoughtful partnership with our patients paired with strategic investment in services such as Acute Care Surgery, we can achieve the aforementioned goal of health equity, specifically for surgical disease. By reframing the value proposition for cholecystectomy from the vantage point of the vulnerable populations who necessarily use Safety-Net Hospitals for care, we can fundamentally change the "Chief Complaint" to a "Chief Context" in which we more fully understand our patients' needs in the context of their lives and provide the timely surgical care that meets their clinical need, while mitigating the Social Determinants of Health that contribute to the need for a Healthcare Safety Net in the first place [3]. It is my fond hope that the authors will replicate their approach across a broad array of clinical conditions so that other disciplines might follow their pioneering path to truly patient-centered care.

\section{References}

1. CMS (2020) Hospital outpatient prospective payment-notice of proposed rulemaking (NPRM) Year 2021. www.CMS.gov. CMS1736-P

2. Hatton GE, Mueck KM, Leal IM et al (2020) Timely care is patient-centered care for patients with acute cholecystitis at a Safety-Net Hospital. World J Surg. https://doi.org/10.1007/ s00268-020-05764-z

3. Dickman SL, Himmelstein DU, Woolhandler S (2017) Inequality and the health-care system in the USA. Lancet 389:1431-1441

Publisher's Note Springer Nature remains neutral with regard to jurisdictional claims in published maps and institutional affiliations. 